\title{
An UNA-Based Approach to Support Mobility in the Internet
}

\author{
Mahnhoon Lee ${ }^{1}$ and Yongik Yoon ${ }^{2}$ \\ ${ }^{1}$ Department of Computing Science, Thompson Rivers University, \\ 900 McGill Rd, Kamloops, BC, V2C5N3 Canada \\ mlee@cariboo.bc.ca \\ ${ }^{2}$ Department of Multimedia Science, Sookmyung Women's University, \\ 53-12 Chungpadong 2Ka, Yongsanku, Seoul, 140-742 Korea \\ yiyoonasookmyung.ac.kr
}

\begin{abstract}
Mobile IP has been developed to give mobile nodes freedom to move to new locations away from home networks. However, Mobile IPv4 requires the implementation of address binding on all IPv4 hosts, i.e., modification, to prevent the long delay due to the triangular routing problem. The host modification is a huge hindrance to the deployment of mobility support. Meanwhile, a new scalable interoperability architecture called UNA (Universal Network Adaptation), which helps the Internet smoothly evolve to MTI (Multi-Tier Internet) or permits easy deployment of a new network technology, was presented. In this paper, we extend UNA, so that we can move the point of address binding from IPv4 hosts to UNA gateways. Therefore, mobility support with no triangular routing problem can be achieved in MTI, without host modification.
\end{abstract}

\section{Introduction}

IPv4 started up without any consideration of mobility support. Mobility support, MIPv4 (Mobile IPv4) [5] and MIPv6 (Mobile IPv6) [1], was recently considered and has been developed. When a mobile node $(\mathrm{MN})$ moves into another new location and is assigned a new address, the address binding to the new address should occur to keep the on-going communications between the $\mathrm{MN}$ and the corresponding nodes (CNs). The address binding could take place at CNs or at a home agent (HA) of the $\mathrm{MN}$, which provides CNs with the location service for MNs. When the address binding takes place at a HA, the traffic route between the $\mathrm{MN}$ and the $\mathrm{CN}$ becomes longer, and it may cause inability to provide good quality streaming services. The problem is called the triangular routing problem.

However, almost all IPv4 hosts, i.e., CNs, on the Internet do not support address binding, because IPv4, unlike IPv6, started without consideration of mobility support. Therefore, in order to solve the triangular routing problem in MIPv4, the address binding should be implemented on every IPv4 host, as explained in [6]. But the deployment of address binding on IPv4 hosts has been delayed, because the modification of hosts is required. As a result, mobility support in the Internet has not been used widely.

Meanwhile, UNA [4] was presented to provide accessibility from IPv4 realms to any other realm, such as IPv6, MTAII [2] and private IPv4. UNA reorganizes the 
subscriber networks of the current IPv4 Internet into LASes (Local Autonomous System) by connecting them to the core network infrastructure through UNA gateways. Later, in [3], UNA was extended in MTI to provide the interoperability among any kind of LASes. Any IPv4-friendly realm, such as private IPv4, public IPv4, IPv6 realms, could be organized as LASes. Hosts in two different LASes can communicate with their own internetworking protocol, IPv4 or IPv6, without any host modification.

In this paper, we explain how to place the point of address binding at UNA gateways rather than hosts, so that we can solve the triangular routing problem without any modification of hosts for easy deployment of mobility support.

\section{UNA-Based Mobility Support}

\subsection{When a CN Initiates a Communication to a MN}

When a $\mathrm{CN}$ initiates a communication, a UNA tunnel is established between LASes. (Refer to [3].) If the peer host is a MN, then the home UNA gateway of the MN sends a bind update message to the UNA gateway of the $\mathrm{CN}$ at steps 3 and 4 in Figure 1. At step 5 , the UNA gateway of the $\mathrm{CN}$ and the foreign UNA gateway of the MN exchange network specific addresses for the $\mathrm{CN}$ and the $\mathrm{MN}$, as shown in [3]. At step 6, the foreign UNA gateway of the MN sends to the MN the global address of the CN's UNA gateway and the network specific address for the $\mathrm{CN}$. Then the MN keeps the addresses for a while for the later handoff. At step 7, the $\mathrm{CN}$ receives the network specific address for the $\mathrm{MN}$, as shown in [3]. Then, the $\mathrm{CN}$ and the $\mathrm{MN}$ can exchange traffic with the network specific addresses decided in the previous steps, as shown in [3].
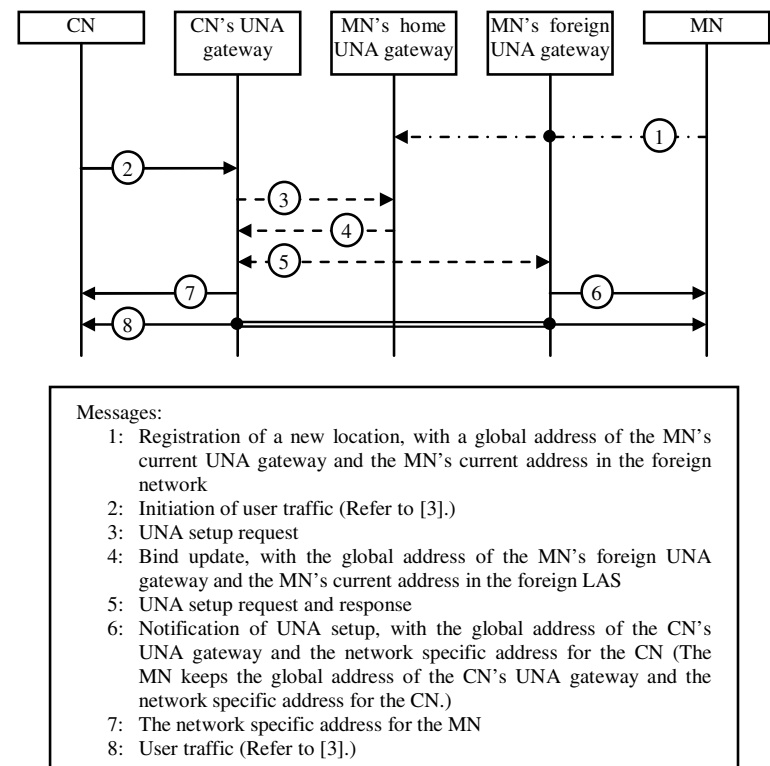

1: Registration of a new location, with a global address of the MN's current UNA gateway and the MN's current address in the foreign network

2: Initiation of user traffic (Refer to [3].)

3: UNA setup request

4: Bind update, with the global address of the MN's foreign UNA gateway and the MN's current address in the foreign LAS

5: UNA setup request and response

6: Notification of UNA setup, with the global address of the CN's UNA gateway and the network specific address for the $\mathrm{CN}$ (The MN keeps the global address of the CN's UNA gateway and the network specific address for the $\mathrm{CN}$.)

7: The network specific address for the MN

8: User traffic (Refer to [3].)

Fig. 1. When a $\mathrm{CN}$ initiates a communication to a $\mathrm{MN}$ 


\subsection{When a MN Initiates a Communication to a $\mathrm{CN}$}

This case is very similar to the previous case in Figure 1. The only difference is that when the $\mathrm{CN}$ is also a $\mathrm{MN}$, a notification of UNA setup is also sent to the $\mathrm{CN}$ from the UNA gateway of the $\mathrm{CN}$.

\subsection{Handoff: When a MN Moves into Other LASes}

In Figure 2, at step 2, the MN registers its new location to its home UNA gateway when it moves into other LAS. At step 3, the MN sends a bind update message with the previous and new location information, i.e., the previous and new global addresses of the MN's UNA gateways, and the previous and new address of the MN. (Actually the MN can communicate with several CNs in the same LAS. In that case, the MN sends a bind update message with the information for all CNs. Steps $4-6$ are also applied to all CNs.) At step 4, two UNA gateways decide new network specific addresses for the $\mathrm{MN}$ and the $\mathrm{CN}$. At step 5, the new network specific address of the $\mathrm{CN}$ is sent to the MN. At step 6, then the UNA gateway of the CN performs address binding between the old and new network specific addresses for the MN.

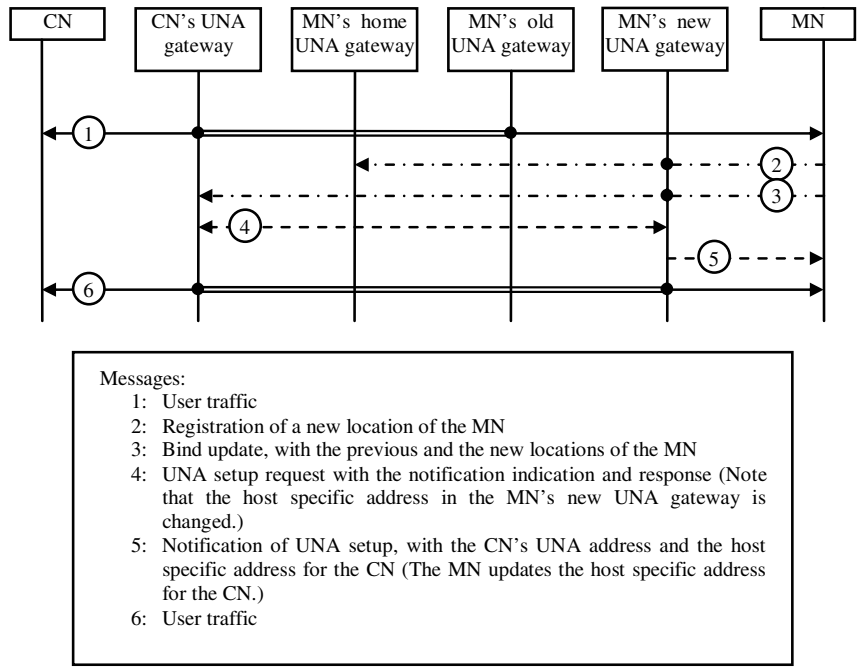

Fig. 2. When a MN moves into another LAS while it is communicating with a $\mathrm{CN}$

\subsection{When a CN Initiates a Communication to a MN, with an Incorrect Network Specific Address for the MN}

The network specific address for the MN will be kept in the LAS of the CN for a while, even after the communication between the $\mathrm{CN}$ and the $\mathrm{MN}$ in Figure 1 finishes. Later the $\mathrm{CN}$ or any other host in the LAS can initiate a communication to the $\mathrm{MN}$ with the same network specific address for the MN. However the MN would 
have moved into other LAS so that the previous foreign UNA gateway no longer has the MN's information for the network specific address.

In the above scenario, the traffic from the $\mathrm{CN}$ will come to the old foreign UNA gateway of the MN. The old foreign UNA gateway of the MN sends a bind update message to the UNA gateway of the CN. The UNA gateway of the CN decides a new network specific address for the $\mathrm{MN}$ as shown at steps $3-6$ in Figure 1 . Then the UNA gateway of the $\mathrm{CN}$ performs address binding between the old and new network specific addresses for the $\mathrm{CN}$ until the old network specific address expires.

\section{Concluding Remarks}

We extend UNA in this paper to move the point of address binding for mobility support in MTI to UNA gateways from hosts or home agents, so that we could solve the triangular routing problem, with a few additional signals. Furthermore, UNA does not require any modification of hosts, and consequently neither does the extension of UNA, allowing for easy deployment.

The experimental implementation of the extension of UNA in MTI to support mobility is on going. In the future, we are planning to investigate mechanisms of fast handoff and group mobility using UNA on MTI, applying to WLAN environments.

\section{References}

1. D. Johnson, C. Perkison and J. Arkko, "Mobility Support in IPv6," Internet-Draft draft-ietfmobileip-ipv6-24.txt (work in progress), June 30, 2003

2. Mahnhoon Lee, "MTAII: New Foundation for the Next Generation Internet," IEEE GLOBECOM, December 2003

3. Mahnhoon Lee and Y. Yoon, "MTI: Integration of UNA and MTAII for the Next Generation Internet", EuroNGI NGI, April 2005

4. Mahnhoon Lee and C. Han, "UNA: A Provision of Accessibility from the IPv4 Internet to Other Networks", IEEE CCNC, January 2005

5. C. Perkins, "IP Mobility Support for IPv4," Internet RFC 3344, August 2002

6. C. Perkins and D. Johnson, "Route Optimization in Mobile IP," Internet Draft draft-ietfmobileip-optim-09.txt (work in progress), 2000 